\title{
Structure and Dynamics in the Local Universe: A Workshop in Honour of Brent Tully's 60th Birthday
}

\author{
Joss Bland-Hawthorn ${ }^{\mathrm{A}}$

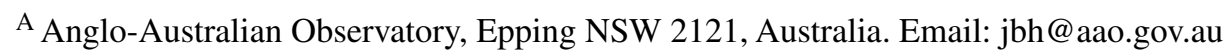

Abstract: We present a summary of a recent meeting held in honour of Brent Tully's 60th birthday. We also provide a retrospective on his career to date.

\section{Workshop Summary}

A group of 45 astronomers convened in Little Bay at Maroubra south of Sydney, Australia, to celebrate the 60th birthday of Brent Tully (see www . aao.gov . au / Tully). The meeting, 'Structure and Dynamics in the Local Universe', focussed on the structure and dynamics of galaxies and the Local Universe, with particular emphasis on new results from the $2 \mathrm{dF}, 6 \mathrm{dF}, 2 \mathrm{MASS}$, SDSS, and HIPASS surveys.

This was an international meeting drawing people from the USA, the UK, Mexico, Germany, Holland, and of course Australia. The participants included Paul Allen, Robbie Auld, Mike Beasley, Kenji Bekki, Joss Bland-Hawthorn, Brian Boyle, Frank Briggs, Sonia Cianci, Matthew Coleman, Matthew Colless, Warrick Couch, Stephane Courteau, Gary da Costa, Jon Davies, Kenton d'Mellow, Marianne Doyle, Michael Drinkwater, Simon Driver, Simon Ellis, Rick Fisher, Ken Freeman,
Brad Gibson, Sebastian Gurovich, Tom Jarrett, Helmut Jerjen, Arna Karick, Baerbel Koribalski, Kelly Kranz, Ofer Lahav, Geraint Lewis, Charlie Lineweaver, Jürgen Ott, Jim Peebles, Dan Pisano, Ivanio Puerari, Emma Ryan-Webber, Elaine Sadler, Will Saunders, Lister Staveley-Smith, Matthias Steinmetz, Jamie Stephens, Brent Tully, Margarita Valdez-Gutierrez, Sylvain Veilleux, Meryl Waugh, and Rachel Webster.

The meeting brought together doctoral students, postdocs, and senior scientists, some of whom have worked with Brent Tully for many years. The theme of the conference tended to concentrate on Brent's more recent work on galaxies, their environments, and the structure of the Local Universe. The review talks introduced the subject matter in each of the workshop sections. Driver talked on luminosity functions and their environs, and questioned their continued use, a theme that was picked up by Jerjen and Briggs. Davies reviewed the progress that

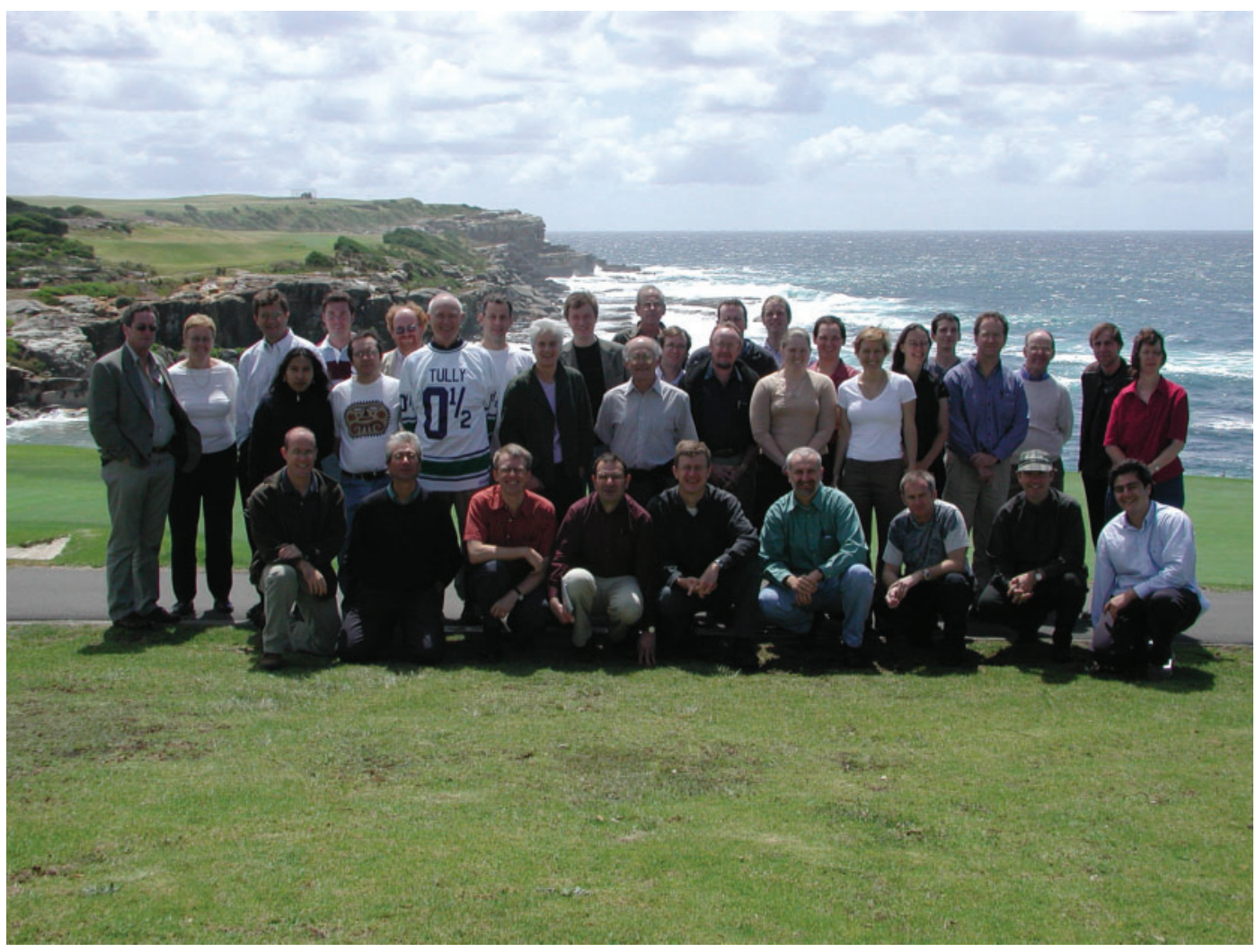


had been made on low luminosity galaxies. Other talks related to dwarf galaxies included Lewis on the recent discovery of the Canis Major dwarf, Drinkwater on his discovery of ultra-compact dwarfs, and Coleman on recently discovered substructure in nearby dwarfs.

Steinmetz spoke on the Tully-Fisher relation richly illustrated with simulations of galaxies. This theme was developed further by Freeman, Gurovich, and Courteau. Peebles presented the cosmic inventory, significantly extending beyond his work with Fukugita and Hogan. Veilleux graphically illustrated the role of galaxy winds and other feedback processes. Saunders gave a comprehensive overview of local supercluster structure and dynamics, and d'Mellow gave the last word on the IRAS dipole. There were memorable reviews of recent surveys including $2 \mathrm{dF}$ (Colless, Lahav), HIPASS (Webster, Staveley-Smith, Pisano), and 2MASS (Jarrett). Tully showed a beautiful simulation of the local structure which brought home the reality of the Local Void. The conference summary and future directions were delivered with distinction by Peebles.

\section{A Retrospective of Brent Tully's Career}

In 2002, Brent Tully was honoured with the Distinguished Graduate Award from the University of Maryland, where in 1974 he graduated with a $\mathrm{PhD}$ for his definitive work on the kinematics of the density waves observations in M51. In order to achieve this, he built an imaging twodimensional interferometer to undertake the most detailed two-dimensional study of motions in any galaxy and provided evidence favouring a model of spiral formation driven by interaction. This work inspired several groups to develop their own Fabry-Perot instruments, in particular, Keith Taylor's development of TAURUS on the AAT and on the WHT, and ultimately the Hawaii Imaging Fabry-Perot Inteferometer (HIFI) on the CFHT and University of Hawaii 2.2-m telescopes.

In the following year, Tully published an Hi study of DDO dwarf galaxies with J. Richard Fisher, someone who was to feature significantly in later years in Tully's research. This program approximately doubled the number of galaxies observed in HI. There followed Westerbork 21-cm observations of what became the smallest systems with detailed kinematic information (with Fisher, Lucette Bottinelli, Lucienne Gouguenheim, Renzo Sancisi, and Hugo van Worden).

In 1977, Tully and Fisher published their most famous work, a luminosity-line width relation for spirals. This is still probably the most accurate way to measure the distances to spiral galaxies beyond a few megaparsecs. In their recent review, Freeman \& Bland-Hawthorn (2002) refer to the enduring mystery of the Tully-Fisher relation which somehow relates dark matter in galaxies to the baryonic content. At the time the relationship was first announced, there was a widespread feeling especially among theorists that the observers had got it wrong as nature could not possibly behave this way.
In 1981, Tully and Fisher published their major HI observations data paper with observations of 1200 galaxies, again approximately doubling the number of galaxies observed in HI at the time. This catalogue was to form the basis for his Nearby Galaxies Catalogue in later years. There are close to 100 papers with Tully-Fisher in the title, a rare distinction indeed. The Tully-Fisher relation has long since passed in to common parlance to the extent that the original reference is only cited in half the papers that refer to the relation.

In later years, Tully became particularly well known for his detailed work on the Local Supercluster, with evidence for de Vaucouleurs' Supercluster Plane and new evidence for the Local Void. This work started with a published description of the Local Supercluster in 1982.

This was a productive time for Tully with papers on the colour-magnitude relation for spirals (with Jeremy R. Mould and the late Marc Aaronson), and a model of galaxy infall toward the Virgo Cluster (with Aaronson, Mould, John P. Huchra, and Paul L. Schechter). There followed a more elaborate model of galaxy infall towards the Virgo Cluster and on the angular momentum content of galaxies (with Edward J. Shaya). In the mid-1970s, Tully coined the expression 'fingers of god' for the linear structures seen in redshift structures associated with massive virialised clusters.

In the mid-1980s, Tully claimed evidence of structure in the Universe on a scale of $0.1 c$. He observed a coincidence of the plane of the Local Supercluster and a plane delineated by Abell and less rich clusters in the PiscesCetus region. Broadly speaking, this structure gains support from the massive $2 \mathrm{dF}$ and Sloan redshift surveys.

In 1986, there was a memorable conference in Kona, Hawaii, organised by Tully and immortalised in Dennis Overbye's 'Lonely Hearts of the Cosmos'. I still recall that meeting well for the exchanges between Gerard de Vaucouleurs and Allan Sandage, for the early results presented by the Seven Samurai, and for the constant sussuration of the Hawaiian surf which came within metres of the beachside lecture room.

It may surprise some that Tully's most cited work is not the famous Tully-Fisher paper from the 1970s. In fact, this honour goes to the the 'Nearby Galaxies Catalog' published in 1987. This is substantially the first attempt to map the structure in the distribution of nearby galaxies. This accompanied the magnificent 'Nearby Galaxies Atlas' (with Fisher). These compendia of nearby groups of galaxies were selected by objective algorithm. He found evidence for dark matter on a scale of $100 \mathrm{kpc}$ around galaxies and lower limit for the density of the Universe of 0.08 times the critical density.

Rounding off a productive decade, Tully published more refined distance estimates to galaxies in clusters (with Mike J. Pierce) and in the field. He also showed evidence that the Hubble Constant is in the range $85-90 \mathrm{~km} \mathrm{~s}^{-1} \mathrm{Mpc}^{-1}$. It was rumoured at this time that he had unsuccessfully applied for a Hawaiian number plate for his car with the registration plate 'H075', so sure was 
he that the number would settle between the two limits promoted by senior figures in the field, a hunch that was ultimately not far adrift from the most plausible value (70 $\mathrm{km} \mathrm{s}^{-1} \mathrm{Mpc}^{-1}$; Freedman et al. 2001).

In 1985, I joined Tully in Hawaii to help set up the HIFI program. This instrument has formed the basis for several outstanding PhDs (e.g. Gerald N. Cecil, Patrick L. Shopbell) and postdocs (Sylvain Veilleux, John Hibbard). The HIFI group produced a series of papers in the 1980s and 1990s of extended emission regions in nearby galaxies with active nuclei. These studies are unrivalled in their quality and detail to the present day. The papers, which continue to be very well cited, provide some of the most compelling evidence for entraining winds driven by starbursts and/or supermassive black holes. These results are discussed in the 2005 Annual Reviews of Astronomy \& Astrophysics on 'Galactic Winds: Near and Far' by Veilleux, Cecil, \& Bland-Hawthorn.

The early 1990s saw Tully back on familiar ground. He began with a linear non-parametric analysis of the velocity field of the Local Supercluster within $3000 \mathrm{~km} \mathrm{~s}^{-1}$ (with Shaya and Pierce). A value for the density of matter clumped on $1-10 \mathrm{Mpc}$ scales of only $0.1 \Omega_{\text {crit }}$ was found. Further intriguing but inconclusive evidence was presented for coherent structure, possibly cyclic, on scales of $300 \mathrm{Mpc}$ (with Roberto Scaramella, Giampaolo Vettolani, and Giovanni Zamorani).

A particularly interesting phase is Tully's Least Action reenactment of orbits of galaxies in the Local Supercluster, which yields a plausible history of the formation of the structure that is seen and provides an estimate of the mass distribution. The density of matter distributed like the observed galaxies corresponds to $17 \%$ of closure density, with a standard deviation of $10 \%$ (with Shaya and Peebles).

In the mid-1990s, Tully and colleague Marc A. W. Verheijen presented evidence for bimodality of the surface brightnesses of spiral disks from optical-infrared photometry of a complete sample of galaxies in the Ursa Major cluster. There may be three discrete formation states for spirals separated by (angular momentum?) thresholds - low surface brightness systems with exponential disks, high surface brightness systems with exponential disks, and high surface brightness systems with disks plus bulges.

By the end of the decade, the extragalactic distance scale and expansion rate are reevaluated by Tully and Pierce with an abundance of new data. Distances to individual galaxies are determined using the relation between luminosities and rotation rates. Samples are now much more extensive and complete, and cover more of the sky to greater distances. Photometry at $B, R, I$, and $K^{\prime}$ bands allows corrections for obscuration. Accurate distances from the cepheid method are available for 24 calibrator galaxies. It is now found that $\mathrm{H}_{0}=77 \pm 8 \mathrm{~km} \mathrm{~s}^{-1} \mathrm{Mpc}^{-1}$ (95\% probability).

In 2001, Tully (with colleagues Rachel S. Somerville, Neil Trentham, and Verheijen) presented observations with the wide field CCD cameras on the CFHT and Subaru telescopes, and complementary Hi observations with the VLA reveal significant variations of the faint end of the galaxy luminosity function in different environments. The ratio dwarf-to-giant is high in high density regions with short dynamical collapse times and low in low density regions with long dynamical times. It is suggested that dwarfs were formed very early in regions with short dynamical times, so early that they formed before the epoch of re-ionisation of the Universe. In low density regions, dwarf dark halos collapsed after the epoch of reionisation. The visible manifestations of galaxy formation were squelched in those halos that were so small that the energy in the ionised gas exceeded the gravitational energy associated with the dark halo potential well.

There is remarkably strong observational evidence for variations in the amount of blue light associated with dark matter in different environments. The most light (lowest $M / L_{B}$ values) are found in group halos in the mass range $10^{12}-10^{13} M_{\odot}$. Collapsed regions on larger mass scales have increasingly larger $M / L_{B}$ values. The correlation is enhanced by a second parameter: groups or clusters with shorter dynamical times have larger $M / L_{B}$ values. These correlations can probably be understood as the consequence of stellar aging, tidal disruptions, and thermalisation of gas components. At smaller masses, there is evidently a cutoff in processes that lead to star formation. Groups in the mass range $10^{11}-10^{12} M_{\odot}$ have very high $M / L_{B}$ values, reaching values of several thousand. It is speculated that there may be completely dark halos. There may be astrophysical mechanisms that prevent gas accumulation and star formation in many low mass halos.

\section{Final Words}

In his summation, Jim Peebles remarked that this meeting reinforced yet again the importance of keeping workshops to about 45 people, the importance of choosing a nice setting, and keeping the scientific content relatively well focussed, all of which were accomplished by this meeting. At the conference dinner, held on the Sydney Harbour, Brent Tully remarked that this was the first meeting where he knew 'what was going on in every talk'.

By all accounts, this was a remarkably successful and enjoyable meeting, greatly aided by the well organised and picturesque conference centre at Little Bay. It is inevitable that there were a number of people (e.g. Shaya, Verheijen, Cecil, Sancisi, Trentham) who were keen to attend but were unable to do so. They, like the conference participants, would want to wish Brent well in the years ahead. 